WORK IN PROGRESS

\title{
Analysis of 3D virtual avatars for video game experience
}

\author{
Aurora Ruiz-Rodriguez, Marijne Wijkstra, Edwin van Asseldonk
}

Published: 30 November 2021

\begin{abstract}
Avatar personalization may contribute to the engagement in a video game [9]. This exploratory study investigated which features a 3D virtual avatar should have for the user to experience Body Ownership. 10 participants (aged 20 to 30) participated in this study. For each participant three avatars were created based on a single picture of them from the shoulders up. The three software that created the avatars are Avatar $S D K^{1}$, Wolf $3 D^{2}$ and Character Creator $^{3}$. After the avatars were created every participant filled out a questionnaire and rated each avatar according to the features. The features that the participants were asked to rate were skin color, hair color, hair style, shape of the face, personal details (e.g. freckles and moles), body shape, height and clothes. The results show that the participants had the highest level of Body Ownership with the avatar from the Avatar SDK, which was rated the highest on the features skin color and shape of the face. Most of the participants would choose the avatar created by the Wolf 3D software to play a video game with, because it has a more cartoon-like look. When the participants were asked what they thought would be the most important features the highest rated answers were skin color, shape of the face and hair color.
\end{abstract}

\section{Keywords:}

3D Virtual Avatars; Body Ownership; Video Games.

\section{Introduction}

In video games, like exergames, the user can be represented by a $3 \mathrm{D}$ virtual avatar. For the user to identify with his or her avatar, there is a need to personalize the avatar. This personalization may contribute to the engagement of the user [9], which is important, because it will motivate them to play the video game. Personalization can be measured by level of embodiment which can be quantified using the embodiment questionnaire [1]. This questionnaire has six sections including Body Ownership. Body Ownership can be defined as the sense of experiencing that the body (in this case avatar) is the own body. This study focusses on the features that a 3D virtual avatar needs to have for the user to

Ruiz-Rodriguez, Aurora., Wijkstra, M Marijne., Van Asseldonk, Edwin. University of Twente

Enschede, The Netherlands.

a.ruizrodriguez@utwente.nl,m.d.wijkstra@student.utwente.nl,

e.h.f.vanasseldonk@utwente.nl experience a high level of Body Ownership. Furthermore, the participants were asked to rate the features that are the most important to match their physical appearance.

\section{Background}

\subsection{Behavioral changes}

In Kilteni et al.[4] it is shown that the way the user is represented in the virtual world influences the behavior and the progress of the user. In this research the avatar that represents the user doesn't look like the user but looks like either a casually dressed dark-skinned person or a formally dressed light-skinned person. The participants were equally and randomly separated into these two groups. In the virtual world the participant is asked to play on a west-African Djembé. The conclusion was that the group with the users who were represented by the casually dressed dark-skinned person made more progress in their ability to drum than the users that were represented by the formally dressed light skinned person. The change in behavior because of the self-representation is also called the Proteus Effect. This effect was researched by Yee et al.[11], the conclusion was that the self-representation has a lot of influence on the behavior of the user. Users change their behavior according to their avatars. This effect in behavioral changes could be very important for exergames that retrain motor abilities in therapy. If the progress of the user can be influenced by a specific representation of the user, that could be of great influence in the rehabilitation of the patient. A meta-analysis has been done by Ratan et al.[6] and they concluded that the Proteus Effect has a large effect compared to other digital media effect or phenomena.

\subsection{Personalization}

Personalization of the avatar contributes to the motivation and engagement of the user. According to Jung et al. [3] there is a small connection between the perception of some body parts in the avatar and the real physical body even though the part in the avatar is not the active body part in the real physical body. In the research of Waltemate et al.[9] it is shown that increasing the level of personalization of an avatar increases the strength of the variables Body Ownership, Presence and Emotional Response. In this research also the impact of clothing is researched. The hypothesis was that a difference in clothing of the user and the virtual avatar would result in more negative outcomes regarding embodiment. This would mean the participant would feel less embodied by the avatar if the clothes of the avatar differed from the clothes worn by

\footnotetext{
${ }^{3}$ www.reallusion.com/character-creator
}

\footnotetext{
${ }^{2}$ wolf3d.io
} 
the participant at that moment. However, this hypothesis was rejected because there was only a minor difference noticeable.

\subsection{Self-identification}

Watts researched the relationship between avatar personalization, self-esteem and perceived social capital [10]. This was done with World of Warcraft (WoW) players. In the WoW game the personalization is done manually and there are a lot of possibilities when it comes to the character creation. The level of selfidentification with their avatar varies between persons. This will also influence the experiences in the game that is played. Watts concluded that there is a significant positive correlation between the avatar self-identification and the self-esteem in the real world. Klimmt et al. further investigated the identification in video games [5]. Participants were assigned one of two roles. They could either be assigned a shooting character or a racing character in a video game. They demonstrated that the character that the participant was assigned to shaped the players' self-concept. This means that the participants that were assigned a shooting character in the game were stronger associated with military concepts and the participants that were assigned a racing character were stronger associated with racing concepts.

\section{Methods}

\subsection{Recruitment of participants}

Participants were able to participate if they were older than 18 , if they were willing to sign a consent form, provide a picture ("selfie") and fill out a questionnaire. This resulted in 10 female participants, aged from 20 to 30 years old.

Disclaimer: Due to the ongoing pandemic (COVID-19) during the time of the study, the participants that were asked to participate were close to the author's inner circle, this could be the cause of the gender bias.

\subsubsection{Materials}

Each participant made one picture of their face from the shoulders up (Figure 1), which is used for the creation of 3 avatars. The avatars are created with three different software packages. These software packages are the online demo of Avatar SDK, the online demo of Wolf $3 D$ and a downloaded demo from Reallusion Character Creator(CC3). These were chosen because of three arguments. First they allowed creation of the avatars within a minute. Second, the look of the three avatars is different. The avatars from Avatar SDK and Character Creator are resembling a more human-like body and face, where the Wolf 3D creates a more cartoon-like version. The biggest difference between the avatar from Avatar SDK and the Character Creator is that the Character Creator software does not scan the hairstyle of the user. The hairstyle needed to be added manually by the participant. Third, all packages could be used for free. A downside of these software packages is that none of the packages scan the body of the user. This means that the body of the avatar is not created based on the picture. Examples of the avatars created with the software packages Avatar SDK, Wolf 3D and Character Creator based on this picture are shown in Figure 2.

\subsection{Data Collection}

Participants were asked to fill out the questionnaire, for further analysis.

\subsubsection{Questionnaire}

The questionnaire consisted out of four sections (S1-S4), explained below. Section 2, 3 and 4 asked for a 5-point Likert scale and a written explanation of the chosen answers. These questions are based on a paper by Debarba et al. and is used to determine the Body Ownership of the user for each avatar, which consist in 6 sections [1]. In this research only the section for Body Ownership is used due to the fact that the avatars are not moving.

S1. Demographic of the participants: the goal in this section is to understand the diversity within the participants.

S2. Avatar type: holds questions regarding self-identification and the participants' preferences.

S3. Avatar comparison: The goal of rating these features is to see which features contribute the most to the feeling of Body Ownership. Participants rate eight features (skin color, hair color, hairstyle, shape of the face, personal details, body shape, height and clothes) of each avatar, where 1 is the feature that does not match their physical appearance at all and 5 means it matches really well.

S4. Importance of features: The goal in this section is to be able to conclude what features are the most important for the participants and why.

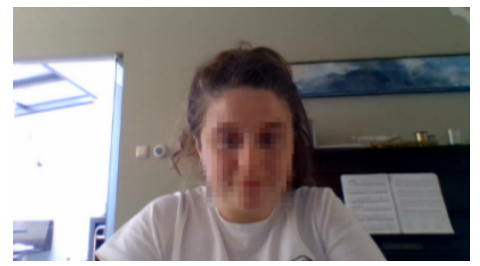

Figure 1. Example of picture for input avatars.

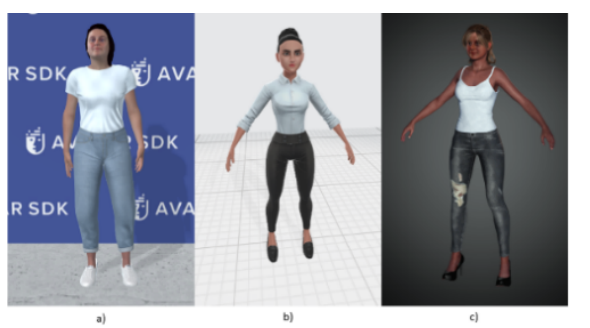

Figure 2. Examples of a)Avatar SDK, b)Wolf 3D avatar, c)Character Creator avatar.

\subsection{Data Analysis}

Using basic statistical techniques, percentages were obtained to show which avatar represents the participant the best according to them and which avatar would be chosen the most for playing a video game. Content analysis was used for the analysis of the qualitative data based on the conceptual content analysis. In conceptual content analysis there are specific labels that are assigned to the text provided by the participants. The labels are skin color, hair color, hair style, face, height, clothes, body shape, general, animation, personal details. Every label also gets a color assigned to it. For example, when a participant talks about the eye color of the avatar, it will get the label personal details. When all the text is labelled, the labels can be counted and therefore the qualitative data can be analysed in a quantitative way. 
Table 1. Results of content analysis of avatars chosen to be best representation.

\begin{tabular}{|l|l|l|}
\hline Participant & $\begin{array}{l}\text { Chosen } \\
\text { Software }\end{array}$ & Codes and Labels \\
\hline P1 & SDK & Clothes, Face $\rightarrow$ realistic \\
\hline P2 & SDK & General $\rightarrow$ realistic \\
\hline P3 & SDK & Face $\rightarrow$ realistic \\
\hline P4 & Wolf 3D & $\begin{array}{l}\text { Hair, skincolor } \rightarrow \text { best } \\
\text { resemblance }\end{array}$ \\
\hline P5 & Wolf 3D & Animation $\rightarrow$ preference \\
\hline P6 & SDK & General $\rightarrow$ most similar \\
\hline P7 & SDK & General $\rightarrow$ best resemblance \\
\hline P8 & SDK & $\begin{array}{l}\text { Face } \rightarrow \text { Exactly physical } \\
\text { appearance }\end{array}$ \\
\hline P9 & SDK & Face $\rightarrow$ best resemblance \\
\hline P10 & CC3 & Face, Details $\rightarrow$ realistic \\
\hline
\end{tabular}

\section{Results}

The avatar from the Avatar $S D K$ was chosen by $70 \%$ of the participants as the avatar that represented them the best, $20 \%$ chose the Wolf $3 D$ avatar and $10 \%$ chose the avatar from Character Creator. The participants were also asked to explain why they made this choice. The results of this analysis is shown in Table 1. The face is mentioned four times as a reason for picking the avatar from Avatar SDK. The face of the avatar from Avatar SDK represents the physical appearance of the participants the best according to these participants. Also the overall look of the avatar is the most realistic for the avatar of Avatar SDK according to P2, P6 and P7.

Interestingly participants would prefer a different avatar if they had to choose the one to use in a game. Of the participants $70 \%$ chose the Wolf $3 D$ for this question, $20 \%$ chose the Avatar SDK and $10 \%$ chose the Character Creator. Furthermore, an explanation for

Table 2. Results of content analysis for which avatar the participants would use in a game.

\begin{tabular}{|l|l|l|}
\hline Participant & $\begin{array}{l}\text { Chosen } \\
\text { Software }\end{array}$ & Codes and Labels \\
\hline P1 & SDK & General $\rightarrow$ best resemblance \\
\hline P2 & CC3 & $\begin{array}{l}\text { General } \rightarrow \text { Resemblance and } \\
\text { preference }\end{array}$ \\
\hline P3 & Wolf 3D & Details $\rightarrow$ pretty \\
\hline P4 & Wolf 3D & General $\rightarrow$ Best re-semblance \\
\hline P5 & Wolf 3D & Animation $\rightarrow$ preference \\
\hline P6 & Wolf 3D & Animation $\rightarrow$ preference \\
\hline P7 & Wolf 3D & $\begin{array}{l}\text { General } \rightarrow \text { Game-look, } \\
\text { Clothes } \rightarrow \text { outfit options }\end{array}$ \\
\hline P8 & Wolf 3D & General $\rightarrow$ Cartoon-look \\
\hline P9 & Wolf 3D & General $\rightarrow$ Game-look \\
\hline P10 & SDK & $\begin{array}{l}\text { General } \rightarrow \text { Realistic but time } \\
\text { efficient }\end{array}$ \\
\hline
\end{tabular}

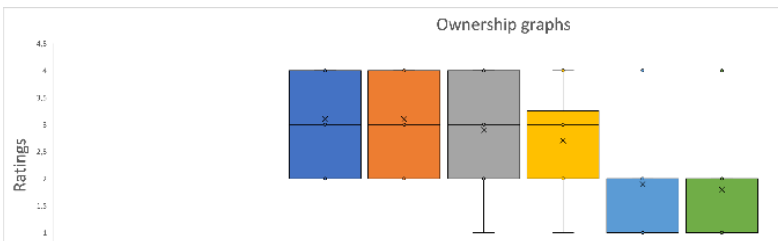

- Avatar SDK: I felt as if I was looking at my own body ( $\mu=3.1 \mathrm{sd}=0.88)$ - Avatar SDK: It felt that the virtual body was my own body $(\mu=3.1 \mathrm{sd}=0.88)$ a Wolf3D : I felt as if I was looking at my own body $(\mu=2.9 \mathrm{sd}=0.99)$ a Wolf 3D: It felt that the virtual body was my own body $(\mu=2.7 \mathrm{sd}=0.95)$ - Character Creator: I felt as if I was looking at my own body ( $\mu=1.9 \mathrm{sd}=0.88$ ) च Character Creator: It felt that the virtual body was my own body $(\mu=1.8 \mathrm{sd}=0.92)$

Figure 4. Boxplot for Body Ownership, $\mathrm{N}=10$.

the choice is asked. The content analysis of this qualitative data is shown in Table 2. The participants like the game-look of the Wolf $3 D$ avatar better when they are playing a game, as evidenced by the answers of P5, P6, P8 and P9. All these participants chose for the Wolf $3 D$ avatar. For example, P7 says: "Because it has a sort of game-look about it, and nice options for outfits".

Participants were also asked what kind of avatar they would like to be represented by. Most participants chose for the option of "An avatar that looks like me" and explained that it would make the game more personal and relatable. The avatar from the Avatar SDK has the highest average rating while the avatar from the Wolf $3 D$ is a little lower. The standard deviation is higher for the Wolf $3 D$ avatar. Figure 4 also shows that the spread of the ratings for the statements of the Wolf $3 D$ is bigger. The avatar from the Character Creator shows the lowest ratings.

The participants were asked to rate each feature on how important it was to match their physical appearance. The boxplots created with the answers of the participants are shown in Figure 5. The features skin color, hair color, shape of the face and body shape rate high in this section. These features also have a small standard deviation, meaning that the ratings of the participants were relatively close to each other. The feature skin color gets the highest rating regarding the importance of the feature. The lowest rating is the one for the clothes. The averages for the features height and clothes are close to each other. However the importance of clothes is valued very differently among participants as evidenced by the high standard deviation and some of the explanations. We can look at the explanation of the participants to understand their answers.

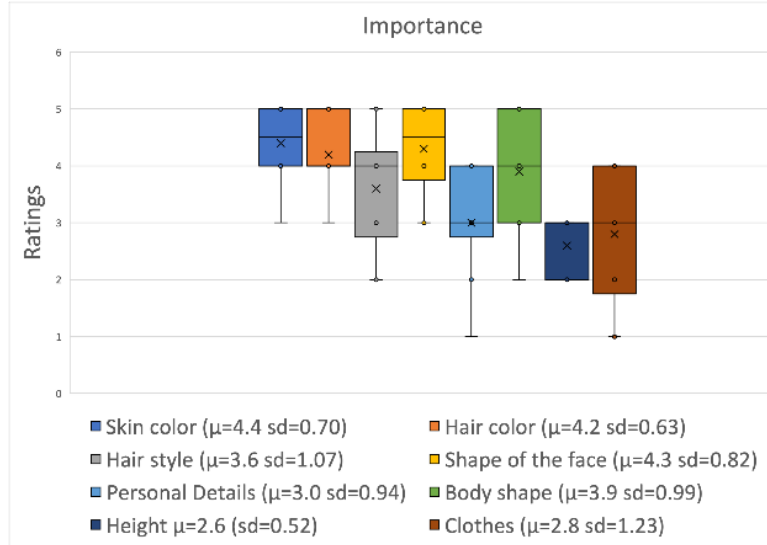

Figure 5. Boxplot for Importance of Features, $\mathrm{N}=10$. 
P10 rated the clothes with a 4 because it is one of the "most important aspects to recognize somebody", however P2 rated the importance of clothes with a 1 because it is "not the same everyday".

\subsection{Discussion}

To measure the Body Ownership for each avatar an existing questionnaire was used. However, this questionnaire was used in experiments where the participants were in a virtual reality with a Head Mounted Display instead of looking at a screen where an avatar is displayed. That means that if they move their head for example to the right, they are still in the same environment and see the other reality. This could have resulted in lower outcomes of these statements for Body Ownership. Another possible cause for the difference between the ratings for Wolf $3 D$ and Avatar SDK on one side and the Character Creator on the other side is that the avatars from Wolf $3 D$ and Avatar SDK could be turned around manually on screen and the avatar from Character Creator could not. This could have increased the feeling of Body Ownership in the Avatar SDK and Wolf $3 D$.

Since Body Ownership is part of the embodiment, the results of this research can be compared to the findings of Waltemate et al. which concluded that there was no negative correlation between a difference in clothing of the avatar and the level of embodiment. The three avatars created for the participant had different clothing than the participant was wearing at that moment since it needed to be adjusted manually and there were limited choices. There have been studies about software that create personalized 3D virtual avatars but these studies miss which features are the most important to match $[2,7,8]$. However, the features skin color, shape of the face and hair color have the highest ratings for importance. This means that for the participants these ratings are the most important to match the physical appearance of the user. The hair color had the highest average rating for the avatar created by the Wolf $3 D$ software.

\section{Conclusion and future work}

Skin color, hair color and shape of the face rated highly on how important these features are for the participants to be able to identify with the avatar. The height, clothes and personal details are all rated low on how important it is for these features to be personalized. Based on these ratings, it can be concluded that the skin color and shape of the face are the most important features for a user to experience Body Ownership with the avatar. An important recommendation for future work is to research the embodiment while moving with a personalized avatar.

\section{Acknowledgments}

This study was part of the project HEROES (Home-based ExeRgaming fOr Enhancing resistance to falls after Stroke).

\section{References}

[1] Henrique Galvan Debarba, Sidney Bovet, Roy Salomon, Olaf Blanke, Bruno Herbelin, and Ronan Boulic. 2017. Characterizing first and third person viewpoints and their alternation for embodied interaction in virtual reality. PLoS

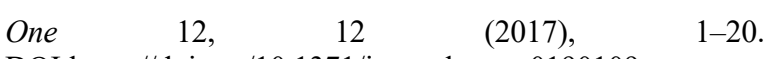

DOI:https://doi.org/10.1371/journal.pone.0190109

[2] Zeng Huang, Yuanlu Xu, Christoph Lassner, Hao Li, and Tony Tung. 2020. ARCH: Animatable Reconstruction of Clothed Humans. Proc. IEEE Comput. Soc. Conf. Comput. Vis. Pattern Recognit. (2020), 3090-3099. DOI:https://doi.org/10.1109/CVPR42600.2020.00316

[3] Sungchul Jung and Charles E. Hughes. 2016. The effects of indirect real body cues of irrelevant parts on virtual body ownership and presence. Int. Conf. Artif. Real. Telexistence Eurographics Symp. Virtual Environ. ICAT-EGVE 2016 (2016), $107-114$ DOI:https://doi.org/10.2312/egve.20161442

[4] Konstantina Kilteni, Ilias Bergstrom, and Mel Slater. 2013. Drumming in immersive virtual reality: The body shapes the way we play. IEEE Trans. Vis. Comput. Graph. 19, 4 (2013), 597-605. DOI:https://doi.org/10.1109/TVCG.2013.29

[5] Christoph Klimmt, Dorothée Hefner, Peter Vorderer, Christian Roth, and Christopher Blake. 2010. Identification with video game characters as automatic shift of selfperceptions. Media Psychol. 13, 4 (2010), 323-338. DOI:https://doi.org/10.1080/15213269.2010.524911

[6] Rabindra Ratan, David Beyea, Benjamin J. Li, and Luis Graciano. 2020. Avatar characteristics induce users' behavioral conformity with small-to-medium effect sizes: a meta-analysis of the proteus effect. Media Psychol. 23, 5 (2020),

$651-675$ DOI:https://doi.org/10.1080/15213269.2019.1623698

[7] Shunsuke Saito, Zeng Huang, Ryota Natsume, Shigeo Morishima, Hao Li, and Angjoo Kanazawa. 2019. PIFu: Pixel-aligned implicit function for high-resolution clothed human digitization. Proc. IEEE Int. Conf. Comput. Vis. 2019Octob, (2019), 2304-2314. DOI:https://doi.org/10.1109/ICCV.2019.00239

[8] Gül Varol, Duygu Ceylan, Bryan Russell, Jimei Yang, Ersin Yumer, Ivan Laptev, and Cordelia Schmid. 2018. BodyNet: Volumetric inference of 3D human body shapes. Lect. Notes Comput. Sci. (including Subser. Lect. Notes Artif. Intell. Lect. Notes Bioinformatics) 11211 LNCS, (2018), 20-38. DOI:https://doi.org/10.1007/978-3-030-01234-2_2

[9] Thomas Waltemate, Dominik Gall, Daniel Roth, Mario Botsch, and Marc Erich Latoschik. 2018. The impact of avatar personalization and immersion on virtual body ownership, presence, and emotional response. IEEE Trans. Vis. Comput. Graph. 24, 4 (2018), 1643-1652. DOI:https://doi.org/10.1109/TVCG.2018.2794629

[10] Melissa Watts. 2016. Avatar Self-Identification, SelfEsteem, and Perceived Social Capital in the Real World: A Study of World of Warcraft Players and their Avatars. March (2016), 76. Retrieved from http://scholarcommons.usf.edu/cgi/viewcontent.cgi?article= $7351 \&$ context $=$ etd

[11] Nick Yee and Jeremy Bailenson. 2007. The proteus effect: The effect of transformed self-representation on behavior. Hum. Commun. Res. 33, 3 (2007), 271-290. DOI:https://doi.org/10.1111/j.1468-2958.2007.00299.x

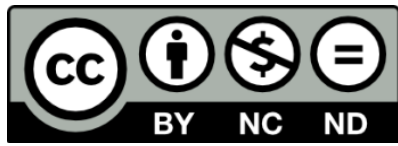

(C) 2021 by the authors. This work is licensed under the Creative Commons AttributionNonCommercial-NoDerivatives 4.0 International License. To view a copy of this license, visit http://creativecommons.org/licenses/by-nc-nd/4.0/ or send a letter to Creative Commons, PO Box 1866, Mountain View, CA 94042, USA. 